\title{
Bifurcation Analysis in a Neuronal Model without/with Delay
}

\author{
Shyan-Shiou Chen
}

\begin{abstract}
In this study, we provide a global picture of the bifurcation scenario of a two-dimensional Hindmarsh-Rose (HR) type model. We present all of the possible classifications based on the following results: first, the number and stability of the equilibrium are analyzed in detail with a table built to show not only how to change the stability of the equilibrium but also which two equilibria collapse through the saddle-node bifurcation; secondly, sufficient conditions for an Andronov-Hopf bifurcation and a saddle-node bifurcation are mathematically confirmed; and finally, we provide sufficient conditions for a Bogdanov-Takens (BT) bifurcation and a Bautin bifurcation. Finally, we present characteristic equation for the HR type model with delay. These results provide us a diversity of behaviors for the model. The results in the paper should be helpful when choosing suitable parameters for fitting experimental observations.
\end{abstract}

Index Terms - Two-dimensional hindmarsh-rose type model, bifurcations.

\section{INTRODUCTION}

In the field of neuroscience [1]-[6] researchers have constructed a variety of different types of biological neural networks. Most of these modeling processes are constructed from experimental observations. To understand whether these constructed neural networks have a richer behavior, many researchers have explored network's basic system properties.

Hindmarsh and Rose (HR) [1]-[3] constructed a model of a bursting neuron from a thalamocortical neuron model with detailed ionic currents, which can be seen as a generalization of the FitzHugh-Nagumo (FN) equations [4], [5], which are a polynomial model and mimic most of the behaviors of the Hodgkin-Huxley equations [6]. The first version was developed by FitzHugh [7] and is a two-dimensional system of ODEs. The main difference between FN and HR is the component of the recovery variable: the former is characterized by the linearity, whereas the latter is described by the quadratic function.

Hodgkin suggested that two different types of neurons, namely, Class I and Class II neurons, exist according to their frequency response characteristics when a constant current is injected into the cell body. Class I neurons go from steady state to oscillatory behavior through a saddle-node bifurcation. Additionally, for Class I neuron, it has been claimed that repetitive firing first appears with zero frequency (homoclinic bifurcation), latency may be

Manuscript received November 20, 2012; revised January 22, 2013.

S. S. Chen is with the Department of Mathematics, National Taiwan Normal University, Taipei, Taiwan (e-mail: sschen@ntnu.edu.tw). arbitrarily long and intermediate-sized responses (in amplitude) are not possible. For Class II neurons, the spiking is initiated through a (subcritical) Hopf bifurcation. The result leads to the onset of oscillations with a well-defined, non-zero frequency and possibly with small amplitude, and the latency for firing is finite. With respect to the classification based on bifurcation theory, these two types of neurons have also been called Type I and Type II, respectively. Bifurcation methodologies enable us to reduce many biophysically accurate HR type models to a two-dimensional system of ordinary differential equations of the form. It is noteworthy that in a plane system, class I excitability is essentially characterized by the quadratic nonlinearity. To date, many two- and three- dimensional HR models [1], [2], [8]-[10] have been studied. However, most of these papers investigated the bifurcations of the HR model though computer simulations. Therefore, it is important to mathematically analyze the $\mathrm{AH}$ and $\mathrm{SN}$ bifurcations of the HR model with bifurcation theory.

In this paper, we present bifurcation diagrams that explain all of the possible behaviors. The paper is organized as follows: In Section II, a 2 -dimensional Hindmarsh-Rose (2DHR) type model proposed with delay is introduced. In Section III, we discuss the number of an equilibrium and its stability for no delay case. For certain conditions, different categories are represented by pictures. Each picture is divided into several zones. We assign to each zone a letter and set out in detail the number of equilibria and their stability in a table. According to this table, we can clearly understand how the number of the solution and its stability changes with the current parameters. In Section IV, the conditions of SN bifurcations are given. we also give an example of a saddle-node bifurcation on a limit cycle. In Section V, the AH and Bautin bifurcations are analyzed. A numerical simulation with bistable behavior is given. In Section VI, we analyze the Bogdanov-Takens bifurcation. In Section VII, we will present the characteristic equation of the model with delay and analyze it's eigenvalues of purely imaginary roots. Finally, the conclusions are presented.

\section{A Two-Dimensional HindMARsh-Rose TyPE MODEL}

Let us consider a two-dimensional Hindmarsh-Rose (2DHR) type model of the following form:

$$
\begin{gathered}
\dot{x}(t)=c\left[k x(t)-x^{3}(t) / 3-y(t)+\right. \\
\left.(1-k)\left(x(t-\tau)-v_{0}\right)+I\right], \\
\dot{y}(t)=\left(x^{2}(t)+d x(t)-b y(t)+a\right) / c
\end{gathered}
$$

where the prime denotes differentiation with respect to the 
independent variable $t$ and the two variables $x$ and $y$ denote the cell membrane potential and a recovery variable, respectively. The parameters $a, b, c, d, \tau$ and $k$ are positive. The parameter $v_{0}$ means the resting potential. The parameter $c$ represents the time scale. The parameter $I$ denotes the membrane current or external stimulus. Because the effect of a nonzero $I$ can be studied by the parameter $a$, we can assume that $I=0$.

\section{EQUILIBRIA AND THEIR STABILITY}

In this section, we attempt to discuss an equilibrium and its stability for Eq. (1) for $k=1$ and $\tau=0$. The study of an equilibrium for Eq. (1) is equivalent to the study of the zero solution of the following cubic function:

$$
h(x)=(b / 3) x^{3}+x^{2}+(d-b) x+a
$$

with $b \neq 0$. We will make clear the existence and the number of zero solutions and analyze their stability of equilibria.

Define

$$
x_{\ell}^{c}=(-1-\sqrt{D}) / b, x_{\mathrm{r}}^{c}=(-1+\sqrt{D}) / b
$$

with $D=1+b^{2}-b d$ and $b \neq 0$. For a positive number $D$, these variables are the right and left critical numbers of $h(x)$, respectively. Obviously, $x_{\ell}^{c}<x_{\mathrm{r}}^{c}$. By $x_{\ell}^{c}, x_{\mathrm{r}}^{c}$, and the sign of $D$, the number of zero solutions can be analyzed. If $D \leq 0$, the cubic function $h$ is monotone. Therefore, there exists only one zero solution. In other words, there exists only one equilibrium for Eq. (1). Let $D>0$. The two critical numbers $x_{\ell}^{c}$ and $x_{\mathrm{r}}^{c}$ exist. If zero is between $h\left(x_{\ell}^{c}\right)$ and $h\left(x_{\mathrm{r}}^{c}\right)$, there exist three equilibria for Eq. (1). If either $h\left(x_{\ell}^{c}\right)=0$ or $h\left(x_{\mathrm{r}}^{c}\right)=0$, there exist two equilibria for Eq. (1). If none of previous two cases holds, only one equilibrium for Eq. (1) exists. Let the notation $(\mathrm{SN})^{ \pm}$denote a triple of parameters $(a, b, d)$ that satisfies the following relation: $(\mathrm{SN}) \pm: a=(1-3 D \pm 2 D \sqrt{D}) /\left(3 b^{2}\right)$. If the condition $(\mathrm{SN})+[$ resp. $(\mathrm{SN})-]$ holds, then $h\left(x_{\mathrm{r}}^{\mathrm{c}}\right)=0$ [resp. $h\left(x_{\ell}^{\mathrm{c}}\right)=0$ ]. Therefore, the existence and the number of equilibria are already analyzed.

Next, the eigenvalues of an equilibrium for a plane system will be discussed. In the two-dimensional case, the linear part $L_{0}$ of Eq. (1) at an equilibrium $\mathbf{x}_{0}=\left(x_{0}, y_{0}\right)$ is a $2 \times 2$ matrix, as follows:

$$
L_{0}=\left[\begin{array}{cc}
c-c x_{0}^{2} & -c \\
\left(2 x_{0}+d\right) / c & -b / c
\end{array}\right] \text {. }
$$

Let $\delta\left(x_{0}\right)$ be the trace of $L_{0}$ and $\Delta\left(x_{0}\right)$ be the determinant of $L_{0}$, where functions $\delta$ and $\Delta: R \rightarrow R$ are as follows:

$$
\begin{gathered}
\delta\left(x_{0}\right)=-c x_{0}^{2}+c-b / c, \\
\Delta\left(x_{0}\right)=b x_{0}^{2}+2 x_{0}-b+d .
\end{gathered}
$$

The characteristic polynomial of $L_{0}$ is $\lambda^{2}-\delta\left(x_{0}\right) \lambda+\Delta\left(x_{0}\right)$. Comparing Eq. (2) with $\Delta$, we notice that $h^{\prime}(x)=\Delta(x)$ for all $x$. The zero solutions of $\Delta$ are $x_{\ell}^{c}$ and $x_{\mathrm{r}}^{c}$. If the first component of an equilibrium $\mathbf{x}_{0}$ is either $x_{\ell}^{c}$ or $x_{\mathrm{r}}^{c}$, then at least one of the eigenvalues is zero. Refer to Condition $(\mathrm{SN})^{ \pm}$. Let $c^{2}>b$. The zero solutions of $\delta$ are $x_{\ell}^{0}=-m_{0}$ and $x_{\mathrm{r}}^{0}=m_{0}$, where $m_{0}=\sqrt{1-b / c^{2}}$, and obviously, $x_{\ell}^{0}<x_{\mathrm{r}}^{0}$. If the first component of an equilibrium $\mathbf{x}_{0}$ is either $x_{\ell}^{0}$ or $x_{\mathrm{r}}^{0}$, then the sum of their eigenvalues is zero. In other words, it is a necessary condition that the eigenvalues are pure imaginary. Let the notation $(\mathrm{AH})^{ \pm}$ denote a quadruple of parameters $(a, b, c, d)$ that satisfies the following relations:

$$
\begin{aligned}
(\mathrm{AH})^{ \pm}: c^{2} & >b \text { and } \\
a & = \pm\left(d-\frac{2 b}{3}-\frac{b^{2}}{3 c^{2}}\right) m_{0}-m_{0}^{2}
\end{aligned}
$$

where $(\mathrm{AH})^{+}$and $(\mathrm{AH})^{-}$are determined by equations $h\left(x_{\ell}^{0}\right)=0$ and $h\left(x_{\mathrm{r}}^{0}\right)=0$, respectively. With the help of the theorem [11], the stability of an equilibrium in the plane can be concluded as follows: If $\Delta\left(x_{0}\right)>0$ and $\delta\left(x_{0}\right)>0$, then $\mathbf{x}_{0}$ is an unstable node. If $\Delta\left(x_{0}\right)>0$ and $\delta\left(x_{0}\right)<0$, then $\mathbf{x}_{0}$ is a stable node. If $\Delta\left(x_{0}\right)<0$, then $\mathbf{x}_{0}$ is a saddle point. If $\Delta\left(x_{0}\right)=0$ and $\delta\left(x_{0}\right)<0$, then there are zero and negative eigenvalues. If $\Delta\left(x_{0}\right)=0$ and $\delta\left(x_{0}\right)=0$, then there are double zero eigenvalues. If $\Delta\left(x_{0}\right)=0$ and $\delta\left(x_{0}\right)>0$, then there are zero and positive eigenvalues. Although these criteria are given, the number of equilibria and their stability are unknown for a parameter-given HR type model. We still need to know the relative position of $x_{0}$ among $x_{\ell}^{0}, x_{\mathrm{r}}^{0}, x_{\ell}^{c}$ and $x_{\mathrm{r}}^{c}$. Consequently, we will separate the parameter domain into several parts. First of all, several lemmas will be given.

Lemma 1. Let $c^{2}>b$ and $D>0$.

1) If $0<b \leq 1$, then $\frac{-1}{b}<x_{\ell}^{0}$.

2) If $1<b$ and $\sqrt{b}<c \leq b^{3 / 2} / \sqrt{b^{2}-1}$, then $\frac{-1}{b} \leq x_{\ell}^{0}$.

3) If $1<b$ and $b^{3 / 2} / \sqrt{b^{2}-1}<c$, then $x_{\ell}^{0}<\frac{-1}{b}$.

By Lemma 1, their relationship between $-1 / b$ and $x_{\ell}^{0}$ is confirmed. The number $-1 / b$ or zero is the middle point between $x_{\ell}^{c}$ and $x_{\mathrm{r}}^{c}$ or $x_{\ell}^{0}$ and $x_{\mathrm{r}}^{0}$, respectively. Because $b>0$, the number $-1 / b$ is always less than zero. Notably, if

$$
c=b^{3 / 2} / \sqrt{b^{2}-1}
$$


then $x_{\ell}^{0}=-1 / b$. The interfaces of these conditions in Lemma 1 are shown in Fig. 1, where the region $\Omega_{2}$ or $\Omega_{1}$ satisfies Condition (a) or (b), respectively, and Condition (c) is represented by both regions $\Omega_{3}$ and $\Omega_{4}$. None of the three conditions is represented by the region $\Omega_{5}$.

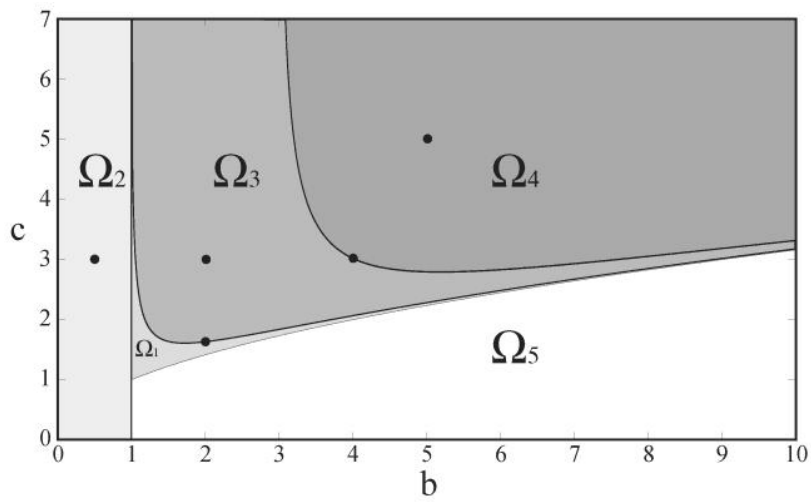

Fig. 1. Illustration of the conditions of Lemma 1 in the parameter domain ( $b, c$ ). Regions $\Omega_{2}$ and $\Omega_{1}$ correspond to Condition (a) and (b), respectively, in Lemma 1. Regions $\Omega_{3}$ and $\Omega_{4}$ correspond to Condition (c) and (b), respectively in Lemma 1 . The relationship between $b$ and $c$ in Region $\Omega_{5}$ satisfies an inequality $c^{2}<b$.

Next, we will discuss the relationship among $x_{\ell}^{0}, x_{\mathrm{r}}^{0}, x_{\ell}^{c}$ and $x_{\mathrm{r}}^{c}$. Let two critical numbers $\hat{L}_{b, c}$ and $L_{b, c}$ be defined as follows:

$$
\begin{aligned}
& \hat{L}_{b, c}=\left[b^{2}+2 c^{2} \sqrt{\left(-b+c^{2}\right) /\left(c^{2}\right)}\right] /\left(c^{2}\right), \\
& L_{b, c}=\left[b^{2}-2 c^{2} \sqrt{\left(-b+c^{2}\right) /\left(c^{2}\right)}\right] /\left(c^{2}\right) .
\end{aligned}
$$

Lemma 2. Consider the following conditions with $c^{2}>b$ and $D>0$.

- Let $x_{\ell}^{0} \leq-1 / b$.

1) If $\hat{L}_{b, c} \leq d$, then $x_{\ell}^{0} \leq x_{\ell}^{c}<x_{\mathrm{r}}^{c}<x_{\mathrm{r}}^{0}$.

2) If $L_{b, c}^{\prime}<d<\hat{L}_{b, c}$, then $x_{\ell}^{c}<x_{\ell}^{0}<x_{\mathrm{r}}^{c}<x_{\mathrm{r}}^{0}$.

3) If $d \leq L_{b, c}^{\prime}$, then $x_{\ell}^{c}<x_{\ell}^{0}<x_{\mathrm{r}}^{0} \leq x_{\mathrm{r}}^{c}$.

- Let $x_{\ell}^{0}>-1 / b$.

1) If $\hat{L}_{b, c} \leq d$, then $x_{\ell}^{c}<x_{\mathrm{r}}^{c} \leq x_{\ell}^{0}<x_{\mathrm{r}}^{0}$.

2) If $L_{b, c}^{\prime}<d<\hat{L}_{b, c}$, then $x_{\ell}^{c}<x_{\ell}^{0}<x_{\mathrm{r}}^{c}<x_{\mathrm{r}}^{0}$.

3) If $d \leq L_{b, c}^{\prime}$, then $x_{\ell}^{c}<x_{\ell}^{0}<x_{\mathrm{r}}^{0} \leq x_{\mathrm{r}}^{c}$.

Next, for a pair of given parameters $(b, c)$, the parameter domain $(a, d)$ will be divided into several regions. For every region, there are different numbers and stability of equilibria for these regions. This basic category is based on the stability and numbers of equilibria. We analyze under which parameter the four conditions $(\mathrm{AH})^{ \pm},\left(\mathrm{SN}^{ \pm}\right)$can be equal. Let the parameters $b, c$ and $d$ satisfy both $(\mathrm{AH})^{+}$and $(\mathrm{AH})^{-}$. In other words, we have the following equation:
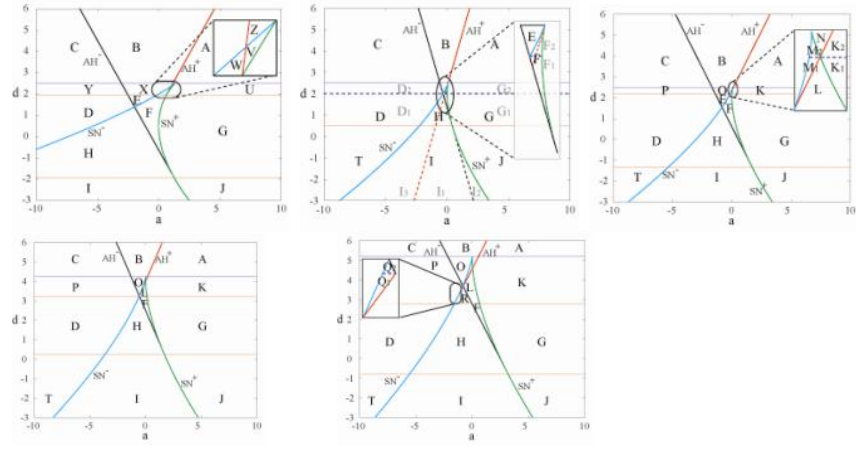

Fig. 2. Illustration of bifurcations with different parameters $b$ and $c$. The horizontal red line above and that below represents $\hat{L}_{b, c}$ and $L_{b, c}$, respectively. The horizontal blue line denotes the line with $d=b+1 / b$ The remaining four non-horizontal curves denote $\mathrm{AH}^{ \pm}$and $\mathrm{SN}^{ \pm}$. The number and stability of equilibria in these partitions with different capital letters can be found in Table I.

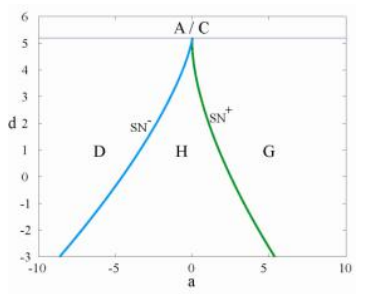

Fig. 3. Illustration of a bifurcation with $c^{2}<b$, where the two parameters $b=5, c=1$ are fixed. The blue horizontal line means $d=b+1 / b$ The other two curves are $\mathrm{SN}^{ \pm}$. The number and stability of equilibria in these partitions with different capital letters can be referred in Table I

Theorem 1. Let two positive parameters $b$ and $c$ with $c^{2}>b$ be given. For the parameter domain $(a, d)$ in Fig. 2, the number of equilibria and their stabilities are listed for different regions in Table I.

Remark. For the positive parameters $b$ and $c$ with $c^{2}<b$, we plot a bifurcation diagram with $b=5$ and $c=1$ in Fig. 3.

TABLE I: ILLUSTRATION OF THE NUMBER OF EQUILIBRIA AND THEIR STABILITY FOR DIFFERENT REGIONS.

\begin{tabular}{cccc}
\hline \hline Region & state & Region & state \\
\hline A,K,G,J & $(\mathrm{s}, \mathrm{N}, \mathrm{N})$ & $\mathrm{F}$ & $(\mathrm{s}, \mathrm{sd}, \mathrm{u})$ \\
$\mathrm{B}, \mathrm{O}, \mathrm{E}, \mathrm{X}$ & $(\mathrm{N}, \mathrm{N}, \mathrm{u})$ & $\mathrm{G}$ & $(\mathrm{s}, \mathrm{N}, \mathrm{N})$ \\
$\mathrm{C}, \mathrm{D}, \mathrm{P}, \mathrm{T}$ & $(\mathrm{N}, \mathrm{N}, \mathrm{s})$ & $\mathrm{H}, \mathrm{I}, \mathrm{R}$ & $(\mathrm{s}, \mathrm{sd}, \mathrm{s})$ \\
$\mathrm{I}$ & $(\mathrm{s}, \mathrm{sd}, \mathrm{s})$ & $\mathrm{M}$ & $(\mathrm{u}, \mathrm{sd}, \mathrm{u})$ \\
$\mathrm{L}$ & $(\mathrm{s}, \mathrm{sd}, \mathrm{u})$ & $\mathrm{N}$ & $(\mathrm{u}, \mathrm{N}, \mathrm{N})$ \\
$\mathrm{Q}$ & $(\mathrm{u}, \mathrm{sd}, \mathrm{s})$ & $\mathrm{U}$ & $(\mathrm{s}, \mathrm{N}, \mathrm{N})$ \\
V & $(\mathrm{s}, \mathrm{sd}, \mathrm{s})$ & $\mathrm{W}$ & $(\mathrm{s}, \mathrm{sd}, \mathrm{u})$ \\
$\mathrm{Y}$ & $(\mathrm{N}, \mathrm{N}, \mathrm{s})$ & $\mathrm{Z}$ & $(\mathrm{N}, \mathrm{N}, \mathrm{s})$ \\
\hline \hline
\end{tabular}

These regions are marked in Fig. 2 and Fig. 3. The capital letters in the same column have the same status. " $\mathrm{N}$ " denotes no equilibrium point. "s" denotes a stable node. " $u$ " denotes an unstable node. "sd" denotes a saddle point. For example, $(s, N, N)$ means one stable equilibrium and $(s, s d, u)$ means one stable, one unstable and one saddle equilibrium. The interface between different regions represent a bifurcation parameter. For example, if the parameter $a$ is decreasing from Region A to $\mathrm{B}$, the stability of the equilibrium is changed. 


\section{SADDLE-NODE BIFURCATION}

A saddle-node bifurcation is a collision and disappearance of two equilibria in dynamical systems. In systems generated by autonomous ODEs, this occurs when the critical equilibrium has one zero eigenvalue. This phenomenon is also called fold or limit point bifurcation. A saddle-node homoclinic bifurcation is also referred to as a saddle-node bifurcation on limit cycle. An important case of saddle-node bifurcation in planar ODEs is when the center manifold makes a homoclinic loop. Such a saddle-node homoclinic bifurcation results in the birth of a limit cycle when the saddle-node disappears. The period of this cycle tends to infinity as the parameter approaches its bifurcation value. The following theorem is provided to show the occurrence of a SN bifurcation. Recall that

$$
(\mathrm{SN})^{ \pm}: a=(1-3 D \pm 2 D \sqrt{D}) /\left(3 b^{2}\right) .
$$

Theorem 1. Assume $D>0$. If all of the parameters in Eq. (1) satisfy Condition $(\mathrm{SN})^{ \pm}$, then a SN bifurcation exists, and its equilibrium is

$$
\left(\frac{-1 \pm \sqrt{D}}{b}, \frac{4-3 b d \pm\left(3 b^{2}-4-b(b-d)\right) \sqrt{D}}{3 b^{3}}\right) .
$$

Corollary 1. If $d=b+\frac{1}{b}$ and $a=\frac{1}{3 b^{2}}$, then there exists a cusp bifurcation.

\section{ANDRONOV-HOPF AND BAUTIN BIFURCATION}

In this section, we analyze a $\mathrm{AH}$ bifurcation which describes when a stable or unstable limit cycle appears out of an unstable or stable equilibrium, respectively. Recall that Condition $(\mathrm{AH})^{ \pm}$only implies that the trace of $L_{0}$ is zero. The following theorem will show that eigenvalues of $L_{0}$ are complex.

Theorem 1. Assume that $c^{2}>b$. If all of the parameters in Eq. (1) satisfy Condition (AH) ${ }^{ \pm}$and $d>\frac{b^{2}}{c^{2}} \pm 2 m_{0}$, then a $\mathrm{AH}$ bifurcation exists, and its equilibrium is

$$
\left(\mp m_{0}, \mp\left(\frac{2}{3}+\frac{b}{3 c^{2}}\right) m_{0}\right) \text {. }
$$

Next, a Bautin bifurcation is considered. For a nearby bifurcation parameter, there exist two limit cycles that collide and disappear via a SN bifurcation of periodic orbits. We analyze the bifurcation by the process in Ref. [12].

\section{Theorem 2.}

1) Let a quadruple of parameters $(a, b, c, d)$ satisfy Condition $\mathrm{AH}^{+}$. If $m_{0} \geq 1 / b$, then a Bautin bifurcation occurs if $d=2 b-b^{2} / c^{2}$ and if $c^{2} \neq b^{3} /\left(b^{2}-1\right)$.

2) Let a quadruple of parameters $(a, b, c, d)$ satisfy

Condition $\mathrm{AH}^{-}$. If $m_{0} \geq 0$, then a Bautin bifurcation occurs if $d=2 b-b^{2} / c^{2}$ and if $c^{2} \neq b^{3} /\left(b^{2}-1\right)$.

For an $\mathrm{AH}$ bifurcation, there are two types: one is a subcritical AH bifurcation, and the other is a supercritical $\mathrm{AH}$ bifurcation. Let us consider Case (a) and (b) in Theorem 2. If $d>2 b-b^{2} / c^{2}$, then $\ell_{1}(0)<0$. The $\mathrm{AH}$ bifurcation is supercritical. If $d<2 b-b^{2} / c^{2}$, then $\ell_{1}(0)>0$. The $\mathrm{AH}$ bifurcation is subcritical.

\section{BOGDANOV-TAKENS BIFURCATION}

In this section, a BT bifurcation is analyzed by the process in Ref. [12] and [13]. The normal form of the BT bifurcation is as follows:

$$
\begin{gathered}
\dot{u}=v, \\
\dot{v}=\beta_{1}+\beta_{2} u+u^{2}-u v
\end{gathered}
$$

We attempt to transform Eq. (1) as Eqs. (6) and (7). For the normal form, we have the following three results for bifurcations: if $4 \beta_{1}-\beta_{2}^{2}=0$, the system undergoes a $\mathrm{SN}$ bifurcation; if $\beta_{1}=0$ and $\beta_{2}<0$, the system undergoes a supercritical AH bifurcation; if $\beta_{1}+(6 / 25) \beta_{2}^{2}=o\left(\beta_{2}^{3}\right)$ and $\beta_{2}<0$, the system undergoes a homoclinic bifurcation curve.

Theorem 1.

(a) Assume that the parameter $a$ satisfies Condition $\mathrm{AH}^{+}$ and $d=\hat{L}_{b, c}$. Then

$$
\beta_{1}=\frac{16 c^{4} m_{0}^{4}\left(\lambda_{1}-m_{0} \lambda_{2}\right)}{\left(1-b m_{0}\right)^{3}}, \beta_{2}=-\frac{4 c^{2} m_{0}^{2} \lambda_{2}}{\left(b m_{0}-1\right)^{2}},
$$

where $\quad \lambda_{1}=a-\left(b-c^{2}\right)\left(2 b m_{0}-3\right) /\left(3 c^{2}\right)$ and $\lambda_{2}=d-\left(b^{2} / c^{2}+2 m_{0}\right)$.

(b) Assume that the parameter $a$ satisfies Condition $\mathrm{AH}^{-}$ and $d=L_{b, c}$. Then

$$
\beta_{1}=\frac{16 c^{4} m_{0}^{4}\left(\lambda_{1}+m_{0} \lambda_{2}\right)}{\left(1+b m_{0}\right)^{3}}, \beta_{2}=-\frac{4 c^{2} m_{0}^{2} \lambda_{2}}{\left(b m_{0}+1\right)^{2}},
$$

where

$$
\lambda_{1}=a-\left(c^{2}-b\right)\left(2 b m_{0}+3\right) /\left(3 c^{2}\right)
$$

$\lambda_{2}=d-\left(b^{2} / c^{2}-2 m_{0}\right)$.

To show the utility of the theorem, some numerical results about the existence of a homoclinic bifurcation are given. Let $(b, c, d)=(2,3,2.25283 \cdots)$ be fixed. When $a=-0.0965$, a stable equilibrium and one saddle equilibrium exist. When $a \approx-0.0969993468$, a homoclinic orbit, one stable equilibrium and one saddle equilibrium exist. When $a=-0.09715$, an unstable limit cycle, one stable equilibrium and one saddle equilibrium exist. In particular, there exist bistable behaviors in this case, due to the fact that there exists a bigger stable limit cycle that enclosed the three above states. 
Therefore, we also confirm that there numerically exists a homoclinic bifurcation.

\section{Characteristic Equation For the Delay CASE}

Here, we consider the model with delay case and analyze the characteristic equation with purely imaginary roots. Let the point $\left(x_{0}, y_{0}\right)$ be an equilibrium and $\bar{x}(t)=x(t)-x_{0}$ and $\bar{y}(t)=y(t)-y_{0}$. To keep the notation simple, the variable $\bar{x}$ is replaced of $x$ and equations can be transformed as follows.

$$
\begin{gathered}
\dot{x}(t)=c\left[\left(k-x_{0}^{2}\right) x(t)-y(t)+(1-k) x(t-\tau)\right] \\
-\frac{1}{3} c x^{3}(t)-c x_{0} x^{2}(t), \\
\dot{y}(t)=\left[\left(2 x_{0}+d\right) x(t)-b y(t)\right] / c+x^{2}(t) / c .
\end{gathered}
$$

The associated characteristic equation is

$$
\operatorname{det}\left(\begin{array}{cc}
c\left(k-x_{0}^{2}\right)+c(1-k) e^{-\lambda \tau}-\lambda & -c \\
\left(2 x_{0}+d\right) / c & -b / c-\lambda
\end{array}\right)=0 .
$$

In other words,

$$
\left[\lambda-c\left(k-x_{0}^{2}+(1-k) e^{-\lambda \tau}\right)\right]\left(\lambda+\frac{b}{c}\right)+\left(2 x_{0}+d\right)=0 .
$$

Eq. (3) is simplified as

$$
F(\lambda)+G(\lambda) e^{-\lambda \tau}=0
$$

where $F(\lambda)=\lambda^{2}+a_{1} \lambda+a_{0} \quad$ and $\quad G(\lambda)=b_{1} \lambda+b_{0} \quad$ with $a_{0}=b\left(x_{0}^{2}-k\right)+\left(2 x_{0}+d\right) \quad, \quad a_{1}=b / c-c\left(k-x_{0}^{2}\right) \quad$, $b_{0}=b(k-1)$ and $b_{1}=c(k-1)$. This characteristic equation determines the local stability of an equilibrium. That is, the equilibrium is stable if and only if all the characteristic roots $\lambda$ have negative real parts.

Applying the Lemma in Cooke and Grossman, we obtain the following results. For Eq. (4), we have the following results: if $a_{0}^{2}<b_{0}^{2}$ holds and $\tau=\tau_{n}^{+}$, then Eq. (4) has a pair of purely imaginary roots $\pm i \omega_{+} ;$if $a_{0}^{2}>b_{0}^{2}$, $b_{1}^{2}-a_{1}^{2}+2 a_{0}>0 \quad, \quad\left(b_{1}^{2}-a_{1}^{2}+2 a_{0}\right)^{2}>4\left(a_{0}^{2}-b_{0}^{2}\right) \quad, \quad$ and $\tau=\tau_{n}^{+}$(resp. $\tau=\tau_{n}^{-}$), then Eq. (4) has a pair of imaginary roots $\pm i \omega_{+}$(resp. $\pm i \omega_{-}$); if neither (1) nor (2), and $\tau>0$, then Eq. (4) has no purely imaginary root, where

$$
\begin{gathered}
\omega_{ \pm}^{2}=\frac{1}{2}\left(b_{1}^{2}-a_{1}^{2}+2 a_{0}\right) \\
\pm\left[\frac{1}{4}\left(b_{1}^{2}-a_{1}^{2}+2 a_{0}\right)^{2}-\left(a_{0}^{2}-b_{0}^{2}\right)\right]^{1 / 2}, \\
\tau_{n}^{+}=\frac{1}{\omega_{+}} \cos ^{-1}\left[\frac{b_{0}\left(\omega_{+}^{2}-a_{0}\right)-\omega_{+}^{2} a_{1} b_{1}}{b_{1}^{2} \omega_{+}^{2}+b_{0}^{2}}\right]+\frac{2 n \pi}{\omega_{+}}, \\
\tau_{n}^{-}=\frac{1}{\omega_{-}} \cos ^{-1}\left[\frac{b_{0}\left(\omega_{-}^{2}-a_{0}\right)-\omega_{-}^{2} a_{1} b_{1}}{b_{1}^{2} \omega_{-}^{2}+b_{0}^{2}}\right]+\frac{2 n \pi}{\omega_{-}},
\end{gathered}
$$

where $n=0,1, \cdots$. Denote

$$
\lambda_{k, n}=\alpha_{k, n}(\tau)+i \omega_{k, n}(\tau), k={ }^{\prime \prime}-{ }^{\prime \prime}{ }^{\prime \prime}+" ; n=0,1,2, \cdots,
$$

where the root of Eq. (4) satisfying $\alpha_{-, n}\left(\tau_{n}^{-}\right)=0$, $\omega_{-, n}\left(\tau_{n}^{-}\right)=\omega_{-}$and $\alpha_{+, n}\left(\tau_{n}^{+}\right)=0, \omega_{+, n}\left(\tau_{n}^{+}\right)=\omega_{+}$. If $\tau_{n}^{+}$and $\tau_{n}^{-}$are bifurcation values, we need to verify if the transversality conditions hold. In other words, the following transversality conditions

$$
\frac{d \operatorname{Re}_{-, n}\left(\tau_{n}^{-}\right)}{d \tau}<0, \frac{d \operatorname{Re}_{+, n}\left(\tau_{n}^{+}\right)}{d \tau}>0
$$

are satisfied due to the fact that

$$
\operatorname{sign}\left(\frac{d \operatorname{Re} \alpha_{ \pm, n}\left(\tau_{n}^{ \pm}\right)}{d \tau}\right)=\operatorname{sign}\left(a_{1}^{2}-b_{1}^{2}-2 a_{0}+2 \omega_{ \pm}^{2}\right) .
$$

Under the conditions, the Hopf bifurcation with delay occurs.

\section{CONCLUSION}

For the 2DHR type model (1), the conditions for a AH, SN, BT and Bautin bifurcation are given. The existence and number of equilibria in Eq. (1) are completely studied. Because the system is a planar system, it follows from the analysis of trace and determinant of the linear part that we discuss the stability of the equilibria. By the Conditions in Lemma 1, the parameter domain $(b, c)$ can be divided into three parts, $\Omega_{1}, \Omega_{2}$ and $\Omega_{3}+\Omega_{4}$, which correspond to Condition (a), (b) and (c) in Lemma 1, respectively. Only $\Omega_{5}$ is excluded in Lemma 1 . The order among $x_{\ell}^{0}, x_{\mathrm{r}}^{0}, x_{\ell}^{c}$ and $x_{\mathrm{r}}^{c}$ is confirmed following Lemma 2. Based on the Lemma, we confirm the signs of $\delta\left(x_{0}\right)$ and $\Delta\left(x_{0}\right)$, where $x_{0}$ is the first component of an equilibrium $\left(x_{0}, y_{0}\right)$ for the 2DHR type model (1). The two signs can help us judge the stability of the equilibrium. With all of the categories based on Lemma 1 and 2, we can furthermore explore all of the AN, $\mathrm{SN}$ bifurcations. Furthermore, the normal forms for the BT and Bautin bifurcations based on the results of the $\mathrm{AH}$ and $\mathrm{SN}$ bifurcations are provided in Theorem 2 and 1, respectively. We also notice that there exist bistable behaviors near the BT and Bautin bifurcation. In addition, it is worth mentioning that $\lim _{c \rightarrow \infty} d=2 b$ in Theorem 2, $\lim _{c \rightarrow \infty} a= \pm\left(d-\frac{2 b}{3}\right)-1$ in Condition $(\mathrm{AH})^{ \pm}$, and there is no parameter $c$ in Condition $(\mathrm{SN})^{ \pm}$. Therefore, as $c$ is sufficiently large, all of the bifurcations curves in the parameter domains $(a, b, d)$ are almost the same. In Section VII, with the characteristic equation, the Hopf bifurcation occurs when purely imaginary roots exist and the transversality condition holds. In the study, we have completely shown the global bifurcation scenario for the 2DHR type model, which may be useful in more biophysical studies, for instance, as a guide in modeling studies for 
choosing suitable parameters for fitting the model to qualitatively different types of electro-physiological behaviors.

\section{ACKNOWLEDGMENT}

This work is partially supported by the National Science Council of Taiwan, the National Taiwan Normal University, and the National Center for Theoretical Sciences of R.O.C. in Taiwan.

\section{REFERENCES}

[1] J. L. Hindmarsh and R. M. Rose, "A model of the nerve impluse using two first-order differential equations," Nature, vol. 296, pp. 162-164, 1982.

[2] J. L. Hindmarsh and R. M. Rose, A model of neuronal bursting using three coupled first order differential equations, in Proc. R. Soc. Lond. B Biol, Sci., vol. 221, pp. 87-102, 1984.

[3] R. M. Rose and J. L. Hindmarsh, "The assembly of ionic currents in a thalamic neuron I. the three dimensional model," in Proc. R. Soc. Lond. $B$, vol. 237, pp. 267-288, 1989 .

[4] R. FitzHugh, "Impulses and physiological states in theoretical models of nerve membrane," Biophys, vol. 1, pp. 445-466, 1961.

[5] J. S. Nagumo, S. Arimoto, and S. Yoshizawa, "An active pulse transmission line simulating nerve axon," in Proc. IRE, vol. 50, pp. 2061-2071, 1962.

[6] A. L. Hodgkin and A. F. Huxley, "A qualitative description of membrane current and its application to conduction and excitation in nerve," J. Physiol, vol. 117, pp. 500-544, 1952.

[7] R. FitzHugh, "Mathematical models of threshold phenomena in the nerve membrane," Bull. Math. Biophysics, vol. 17, pp. 257-269, 1955.
[8] G. Innocentia, A. Morelli, R. Genesio, and A. Torcini, "Dynamical phases of the hindmarsh-rose neuronal model: studies of the transition from bursting to spiking chaos," Chaos, vol. 17, 2007.

[9] M. Storace, D. Linaro, and E. de Lange, "The hindmarsh-rose neuron model: Bifurcation analysis and piecewise-linear approximations," Chaos, 2008.

[10] S. Tsuji, T. Ueta, H. Kawakami, H. Fujii, and K. Aihara, "Bifurcations in two-dimensional hindmarsh-rose type model," Int. J. Bifurcation and Chaos, vol. 17, no. 3, pp. 985-998, 2007.

[11] R. C. Robinson, "An introduction to dynamical systems:Continuous and discrete," Pearson, 2004.

[12] Y. A. Kuznetsov, Elements of Applied Bifurcation Theory, 3rd ed., Number 112 in Applied Mathematical Sciences, New York: Springer Verlag, 2004, ch. 8, pp. 307-324.

[13] F. A. Carrillo, F. Verduzco, and F. Delgado, "Analysis of the takens-bogdanov bifurcation on m-parameterized vector fields," Int. J. Bifurc. Chaos, vol. 20, pp. 995-1005, 2010.

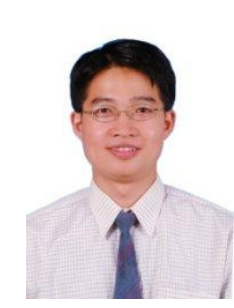

S. S. Chen received the M.S. and Ph.D. degrees in mathematics from National Chiao Tung University, Hsinchu, Taiwan, in 1998 and 2002, respectively. He was a postdoctoral research fellow of the Laboratory of Integrated Brain Research in the Department of Medical Research and Education, Taipei Veterans General Hospital, Taipei, Taiwan from 2003 to 2007 $\mathrm{He}$ is currently an assistant professor of Department of Mathematics, National Taiwan Normal University, Taipei, Taiwan. His research interests include nonlinear time series analysis, synchronization, dynamics of neural networks, qualitative theory of differential equations and difference equations. 